\title{
A GROUP RING METHOD FOR FINITELY GENERATED GROUPS $\left({ }^{1}\right)$
}

\author{
BY \\ KUO-TSAI CHEN
}

It is an essential principle employed in this paper that properties of a group which has only a multiplication can be studied in a ring which has both additive and multiplicative operations. We also achieve, to a certain extent, a unified theory to explain some facts concerning finitely generated groups which are contained in the works of J. W. Alexander, W. Magnus, K. Reidemeister, and R. H. Fox.

A group may be defined by generators and relations; i.e., it may be taken as a factor group $F / R$, where $R$ is a normal subgroup of a free group $F$. H. Hopf [5] has shown that $[F, F] /[F, R]$ is invariantly defined for $F / R$; to be explicit, $F / R \cong F^{\prime} / R^{\prime}$ implies $[F, F] /[F, R] \cong\left[F^{\prime}, F^{\prime}\right] /\left[F^{\prime}, R^{\prime}\right]$. After some preliminaries about group rings in $\$ 1$, we proceed to establish a theory of presentations of groups and to generalize the above mentioned factor group in §3. A family of invariantly defined quotient rings are obtained from the group ring of $J F$ of $F$ over $J$. In $\S 5$, we extract invariants from these quotient rings by mapping each of them homomorphically into another ring of a suitable structure. The homomorphisms we use for this purpose are those induced by a homomorphism of $J F$ into a noncommutative formal power series ring due to Magnus.

A family of groups under our consideration for equivalence by isomorphism of ten have some common properties. For example, in the family of knot groups, each group made abelian is infinite cyclic. Therefore stronger invariants may be expected. Having this in mind, we develop the $B$-presentation theory in $\S 6$. In order to derive invariants from the invariantly defined quotient rings just mentioned, Fox free differentiation becomes our main tool. Invariants resembling Alexander polynomials are produced; the computation for these invariants bears close relation with the Jacobian matrix theory in [4].

$\$ 4$ is mainly concerned with properties of noncommutative formal power series rings.

The following notations will be universal in this paper:

For elements $u_{1}, u_{2}, \cdots$ of a group, $\left[u_{1}, u_{2}\right]=u_{1} u_{2} u_{1}^{-1} u_{2}^{-1}$, and $\left[u_{1}, \cdots, u_{p+1}\right]=\left[\left[u_{1}, \cdots, u_{p}\right], u_{p+1}\right], p \geqq 2$. Let $A$ and $B$ be subgroups of

Presented to the Society, April 25, 1952; received by the editors April 25, 1952 and, in revised form, November 3, 1952.

(1) The major part of the work was done while the author held a research associateship in the Mathematics Department of University of Illinois. 
a group. Then $[A, B]$ denotes the group generated by all $[a, b], a \in A, b \in B$. For any group $G, G_{1}=G, \cdots, G_{p+1}=\left[G_{p}, G\right], \cdots$.

For elements $U_{1}, U_{2}, \cdots$ of a ring, $\left[U_{1}, U_{2}\right]=U_{1} U_{2}-U_{2} U_{1}$, and $\left[U_{1}, \cdots, U_{p+1}\right]=\left[\left[U_{1}, \cdots, U_{p}\right], U_{p+1}\right], p \geqq 2$.

Free groups considered in this paper will be finitely generated.

The author is indebted to R. Baer and J. L. Koszul for valuable suggestions.

1. Group rings. Let $G$ be a multiplicative group, and $J$ a ring with unit element 1 . The group ring $J G$ of $G$ over $J$ consists of the totality of finite formal sums $\sum \lambda_{i} u_{i}, u_{i} \in G, \lambda_{i} \in J$. The addition in $J G$ is formal, and the multiplication is given by $\left(\sum \lambda_{i} u_{i}\right)\left(\sum \mu_{j} v_{j}\right)=\sum_{i, j}\left(\lambda_{i} \mu_{j}\right)\left(u_{i} v_{j}\right)$. Identify $1 \cdot u$ and $u, u \in G$. Then $G$ is contained in $J G$, and the identity $e$ of $G$ is the unit element of $J G$. Furthermore, identify $\lambda e$ and $\lambda, \lambda \in J$; in particular, $1 \cdot e=e=1$. Then $J \subset J G$, and $J G$ is an algebra over $J$.

Let $\rho: G \rightarrow G^{\prime}$ be any homomorphism of the group $G$ into another group $G^{\prime}$. Then $\rho$ can be extended by linearity to a ring homomorphism $\rho: J G \rightarrow J G^{\prime}$ with $\rho(\lambda)=\lambda, \lambda \in J$. The extended homomorphism is also denoted by $\rho$, when there is no possible confusion.

The group ring of the trivial group $\{e\}$ consisting of the identity element only is the ring $J$. The ring homomorphism $o: J G-J$ extended from the trivial homomorphism $G \rightarrow\{e\}$ is called the coefficient homomorphism of $J G$. For any $\sum \lambda_{i} u_{i} \in J G, o\left(\sum \lambda_{i} u_{i}\right)=\sum \lambda_{i}$. The kernel of $o$ is called the fundamental ideal of $J G$ and is generated by all $u-1, u \in G$. If $G$ has a set of generators $\left\{x_{i}\right\}, i \in P$, then $o$ can be regarded also as the ideal generated by all $x_{i}-1, i \in P$.

Let $M$ be a subgroup of $G$, and $N$ a normal subgroup of $M$. Denote by $(N-1)_{M}$ the ideal of the ring $J M \subset J G$ generated by all $u-1, u \in N$.

Lemma 1. Let $M$ and $M^{\prime}$ be subgroups of $G$ and $G^{\prime}$ respectively, and $N$ and $N^{\prime}$ normal subgroups of $M$ and $M^{\prime}$ respectively, while $\sigma_{1}$ and $\sigma_{2}$ are homomorphisms of $G$ into $G^{\prime}$ with $\sigma_{i}(M) \subset M^{\prime}, \sigma_{i}(N) \subset N^{\prime}, i=1$, 2. If $\sigma_{1}$ and $\sigma_{2}$ induce the same homomorphism $M / N \rightarrow M^{\prime} / N^{\prime}$, then the extended homomorphisms $\sigma_{i}: J G \rightarrow J G^{\prime}, \quad i=1,2$, induce the same homomorphism $J M /(N-1)_{M}$ $\rightarrow J M^{\prime} /\left(N^{\prime}-1\right)_{M^{\prime}}$. This implies $\sigma_{1}(u) \equiv \sigma_{2}(u) \bmod \left(N^{\prime}-1\right)_{M^{\prime}}$ for any $u \in J M$.

Proof. First it is clear that $\sigma_{i}(J M) \subset J M^{\prime}$ and $\sigma_{i}\left((N-1)_{M}\right)$ $C\left(N^{\prime}-1\right)_{M^{\prime}}, i=1,2$. Let $u=\sum \lambda_{i} u_{i}$ be any element of $J M$. For each $i$, $\sigma_{1}\left(u_{i}\right) \equiv \sigma_{2}\left(u_{i}\right) \bmod N^{\prime}$. There exists $h_{i} \in N^{\prime}$ with $\sigma_{1}\left(u_{i}\right)=\sigma_{2}\left(u_{i}\right) h_{i}=\sigma_{2}\left(u_{i}\right)\left(h_{i}-1\right)$ $+\sigma_{2}\left(u_{i}\right) \equiv \sigma_{2}\left(u_{i}\right) \bmod \left(N^{\prime}-1\right)_{M^{\prime}}$. Hence $\sigma_{1}(u) \equiv \sigma_{2}(u) \bmod \left(N^{\prime}-1\right)_{M^{\prime}}$, and the lemma is proved.

\section{A lemma.}

Lemma 2. Let $\Re_{1}, \Re_{1}^{\prime}, \Re_{2}, \Re_{2}^{\prime}$ be rings, and $\phi, \phi^{\prime}, \sigma_{i}, \bar{\sigma}_{i}, i=1,2$, ring homomorphisms as given in the following diagram 


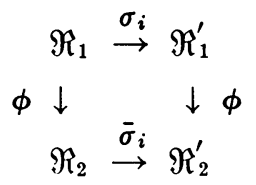

where $\phi$ is onto, and $\phi^{\prime} \sigma_{i}=\bar{\sigma}_{i} \phi, i=1,2$. Let $\mathfrak{N}_{1}, \mathfrak{N}_{1}^{\prime}, \mathfrak{N}_{2}, \mathfrak{N}_{2}^{\prime}$ be ideals in $\mathfrak{R}_{1}, \mathfrak{R}_{1}^{\prime}$, $\Re_{2}, \mathfrak{R}_{2}^{\prime}$, respectively, such that $\phi\left(\mathfrak{N}_{1}\right) \subset \mathfrak{N}_{2}, \phi^{\prime}\left(\mathfrak{N}_{1}^{\prime}\right) \subset \mathfrak{N}_{2}^{\prime}, \sigma_{i}\left(\mathfrak{N}_{1}\right) \subset \mathfrak{N}_{1}^{\prime}, \bar{\sigma}_{i}\left(\mathfrak{N}_{2}\right)$ $\subset \mathfrak{N}_{2}^{\prime}, i=1$, 2. If $\sigma_{1}$ and $\sigma_{2}$ induce the same homomorphism $\mathfrak{R}_{1} / \mathfrak{N}_{1} \rightarrow \mathfrak{R}_{1}^{\prime} / \mathfrak{N}_{1}^{\prime}$, then $\bar{\sigma}_{1}$ and $\bar{\sigma}_{2}$ will induce the same homomorphism $\mathfrak{\Re}_{2} / \mathfrak{N}_{2} \rightarrow \Re_{2}^{\prime} / \mathfrak{N}_{2}^{\prime}$.

Proof. By the hypothesis of the lemma, $\sigma_{1}(u) \equiv \sigma_{2}(u) \bmod \Re_{1}^{\prime}, u \in \Re_{1}$. Every element of $\Re_{2}$ has the form $\phi(u), u \in \Re_{1}$. Then $\bar{\sigma}_{1} \phi(u)=\phi^{\prime} \sigma_{1}(u) \equiv \phi^{\prime} \sigma_{2}(u)$ $\bmod \mathfrak{N}_{2}^{\prime} \equiv \bar{\sigma}_{2} \phi(u) \bmod \mathfrak{N}_{2}^{\prime}$. Hence the lemma is proved.

COROLlary. The lemma also holds if the words "ring" and "ideal" are replaced by "group" and "normal subgroup" respectively.

3. Presentations. A group presented by generators and relations may be regarded as a factor group $F / R$ of a free group $F$. We shall consider necessary conditions, in terms of invariants, that two such groups $F / R$ and $F^{\prime} / R^{\prime}$ are isomorphic. The following lemma is well known and is basic to our theory.

LemmA 3. Given a homomorphism $\rho: F / R \rightarrow F^{\prime} / R^{\prime}$, there exists a homomorphism $\sigma: F \rightarrow F^{\prime}, \sigma(R) \subset R^{\prime}$, such that $\sigma$ induces $\rho$; i.e., $\rho(u R)=\sigma(u) R^{\prime}$, $u \in F$.

Proof. Let $x_{1}, \cdots, x_{m}$ be the free generators of $F$. Choose an element $u_{i}^{\prime}$ from each coset $\rho\left(x_{i} R\right)$. Define $\sigma: F \rightarrow F^{\prime}$ by $\sigma\left(x_{i}\right)=u_{i}^{\prime}, i=1, \cdots, m$. Since $F$ is free, this construction is possible. Then, for any $v=x_{i_{1}}^{\epsilon_{1}} \cdots x_{i_{k}}^{\epsilon_{k}} \in F$, $\sigma(v)=u_{i_{1}}^{\prime \epsilon_{1}} \cdots u_{t_{k}}^{\prime \epsilon_{k}}$ belongs to the coset $\rho(v R)$. Hence $\sigma$ has $\rho$ as its induced homomorphism.

Let $R$ be a normal subgroup in a free group $F$. The pair $\{F / R\}$ is called a presentation. By a presentation homomorphism

$$
\sigma:\{F / R\} \rightarrow\left\{F^{\prime} / R^{\prime}\right\}
$$

we mean a homomorphism $\sigma: F \rightarrow F^{\prime}$ with $\sigma(R) \subset R^{\prime}$. Two such maps $\sigma:\{F / R\} \rightarrow\left\{F^{\prime} / R\right\}$ and $\tau:\{F / R\} \rightarrow\left\{F^{\prime} / R^{\prime}\right\}$ are said to be equivalent if $\sigma$ and $\tau$ induce the same homomorphism $F / R \rightarrow F^{\prime} / R^{\prime}$; i.e., $\sigma(u) \equiv \tau(u) \bmod R^{\prime}$, $u \in F$.

A map $\sigma$ is said to be a presentation isomorphism: $\sigma:\{F / R\} \cong\left\{F^{\prime} / R^{\prime}\right\}$, if $\sigma$ induces an isomorphism-onto $F / R \cong F^{\prime} / R^{\prime}$.

An algebraic invariant $V$ is defined as follows:

(1) $V=V(F, R)$ is a function of $\{F / R\}$, and each $V(F, R)$ is a ring (or group).

(2) Each presentation homomorphism $\sigma:\{F / R\} \rightarrow\left\{F^{\prime} / R^{\prime}\right\}$ defines 
uniquely a ring (or group) homomorphism $\sigma^{*}: V(F, R) \rightarrow V\left(F^{\prime}, R^{\prime}\right)$, called the associated homomorphism of $\sigma$.

(3) If $\sigma$ and $\tau$ are equivalent, then $\sigma^{*}=\tau^{*}$.

(4) For $\sigma_{1}:\{F / R\} \rightarrow\left\{F^{\prime} / R^{\prime}\right\}$ and $\sigma_{2}:\left\{F^{\prime} / R^{\prime}\right\} \rightarrow\left\{F^{\prime \prime} / R^{\prime \prime}\right\}$, we have $\left(\sigma_{2} \sigma_{1}\right)^{*}=\sigma_{2}^{*} \sigma_{1}^{*}$.

(5) If $\sigma:\{F / R\} \cong\{F / R\}$ is the identity isomorphism, i.e., $\sigma(u)=u$, $u \in F$, then $\sigma^{*}: V(F, R) \cong V(F, R)$ is the identity ring (or group) isomorphism.

THEOREM 1. Let $V$ be an algebraic invariant. Then $F / R \cong F^{\prime} / R^{\prime}$ implies $V(F, R) \cong V\left(F^{\prime}, R^{\prime}\right)$.

Proof. Let $\sigma:\{F / R\} \cong\left\{F^{\prime} / R^{\prime}\right\}$ be a presentation isomorphism, and $\rho: F / R \cong F^{\prime} / R^{\prime}$, the homomorphism induced by $\sigma$. There is a presentation isomorphism $\tau:\left\{F^{\prime} / R^{\prime}\right\} \cong\{F / R\}$ such that $\tau$ induces $\rho^{-1}$. Then $\tau \sigma:\{F / R\}$ $\cong\{F / R\}$ induces the identity isomorphism $F / R \cong F / R$. Similarly $\sigma \tau$ induces the identity isomorphism $F^{\prime} / R^{\prime} \cong F^{\prime} / R^{\prime}$. For the associated homomorphisms, we have $(\tau \sigma)^{*}=\tau^{*} \sigma^{*}=1$ and $(\sigma \tau)^{*}=\sigma^{*} \tau^{*}=1$. Hence $\sigma^{*}: V(F, R) \cong V\left(F^{\prime}, R^{\prime}\right)$ is an isomorphism-onto.

A factor-group invariant $M / N$ is an algebraic invariant defined as follows:

(1) Each $M(F, R)$ is a subgroup of $F$, and each $N(F, R)$ is a normal subgroup of $M(F, R)$.

(2) For any $\sigma:\{F / R\} \rightarrow\left\{F^{\prime} / R^{\prime}\right\}$, we have $\sigma(M(F, R)) \subset M\left(F^{\prime}, R^{\prime}\right)$ and $\sigma(N(F, R)) \subset N\left(F^{\prime}, R^{\prime}\right)$. The induced homomorphism $\sigma^{*}: M(F, R) / N(F, R)$ $\rightarrow M\left(F^{\prime}, R^{\prime}\right) / N\left(F^{\prime}, R^{\prime}\right)$ is the associated homomorphism of $\sigma$ with respect to the invariant $M / N$.

The following are a few examples of factor group invariants: (1) $F / R$; (2) $[F, F] /[F, R]$; (3) $R \cap[F, F] /[F, R]$; (4) $F / F_{p} \cdot R$; (5) $F_{p} / F_{p} \cap R$. (1) is obviously a factor group invariant. It follows easily from a result in [5] that (2) and (3) are factor group invariants. Let $G=F / R$. Then $G / G_{p} \cong F / F_{p} \cdot R$ and $G_{p} \cong F_{p} / F_{p} \cap R$. It will not be hard to verify that (4) and (5) are also factor group invariants.

A quotient ring invariant $\mathfrak{M} / \mathfrak{R}$ is an algebraic invariant defined as follows:

(1) Each $\mathfrak{M}(F, R)$ is a subring of $J F$, and each $\mathfrak{R}(F, R)$ is an ideal of $\mathfrak{M}(F, R)$.

(2) Let $\sigma: J F \rightarrow J F^{\prime}$ be extended from $\sigma:\{F / R\} \rightarrow\left\{F^{\prime} / R^{\prime}\right\}$. Then $\sigma(\mathfrak{M}(F, R)) \subset \mathfrak{M}\left(F^{\prime}, R^{\prime}\right)$ and $\sigma(\mathfrak{R}(F, R)) \subset \mathfrak{N}\left(F^{\prime}, R^{\prime}\right)$, and the induced homomorphism $\sigma^{\sharp}: \mathfrak{M}(F, R) / \mathfrak{N}(F, R) \rightarrow \mathfrak{M}\left(F^{\prime}, R^{\prime}\right) / \mathfrak{N}\left(F^{\prime}, R^{\prime}\right)$ is the associated homomorphism of $\sigma$ with respect to the invariant $\mathfrak{M} / \mathfrak{N}$.

An invariant ideal $I$ of a free group ring $J F$ is defined as follows:

(1) $I=I(J F)$ is an ideal of $J F$ and is a function of $J F$.

(2) For any $\sigma: J F \rightarrow J F^{\prime}$ extended from $\sigma: F \rightarrow F^{\prime}$, we have $\sigma(I(J F))$ $\subset I\left(J F^{\prime}\right)$. 
Let $I_{1}$ and $I_{2}$ be invariant ideals, and $\mathfrak{M} / \mathfrak{N}$ a quotient ring invariant. Then $\left(\mathfrak{M} \cap I_{1}+I_{2}\right) /\left(\mathfrak{R} \cap I_{1}+I_{2}\right)$ is called the $\left(I_{1}, I_{2}\right)$-filtration of $\mathfrak{M} / \mathfrak{N}$.

Lemma 4. The $\left(I_{1}, I_{2}\right)$-filtration of $\mathfrak{M} / \mathfrak{N}$ is also a quotient ring invariant.

Proof. Let $\sigma:\{F / R\} \rightarrow\left\{F^{\prime} / R^{\prime}\right\}$ be any presentation homomorphism, and let $\tau$ be equivalent to $\sigma$. Each element of $\mathfrak{M}(F, R) \cap I_{1}(J F)+I_{2}(J F)$ is of the form $u+v, u \in \mathfrak{M}(F, R) \cap I_{1}(J F), v \in I_{2}(J F)$. Since $\mathfrak{M} / \mathfrak{N}$ is a quotient ring invariant, $\sigma(u)-\tau(u) \equiv 0 \bmod \mathfrak{N}\left(F^{\prime}, R^{\prime}\right)$. On the other hand $\sigma(u)-\tau(u)$ $\equiv 0 \bmod I_{1}\left(J F^{\prime}\right)$. Thus $\sigma(u)-\tau(u) \equiv 0 \bmod \mathfrak{N}\left(F^{\prime}, R^{\prime}\right) \cap I_{1}\left(J F^{\prime}\right)$ and $\sigma(u+v)$ $\equiv \tau(u+v) \bmod \mathfrak{N}\left(F^{\prime}, R^{\prime}\right) \cap I_{1}\left(J F^{\prime}\right)+I_{2}\left(J F^{\prime}\right)$. It is easy to verify that the other conditions in the definition of a quotient ring invariant are also satisfied by $\left(\mathfrak{M} \cap I_{1}+I_{2}\right) /\left(\mathfrak{R} \cap I_{1}+I_{2}\right)$. Hence the lemma is proved.

The following is a direct consequence of Lemma 1 :

THEOREM 2. Let $M / N$ be a factor group invariant. Then $J M /(N-1)_{M}$ is a quotient ring invariant.

A part of the conceptions in this section may be found in the works of Baer [2]. The definition of an algebraic invariant is extremely close to that of a functor due to Eilenberg and MacLane [3]. Since a presentation isomorphism does not necessarily possess an inverse, the equivalence (by isomorphism) of presentations does not agree with the equivalence in the sense as given in [3]. We are thus unable to avoid using the notion of algebraic invariants in spite of some repetition.

4. Formal power series. Let $J$ be a ring with unit element 1 , and let $X_{1}, \cdots, X_{m}$ be noncommutative indeterminates. Denote by $\mathfrak{F}_{p}$ the totality of forms $\sum_{1 \leqq i_{1}, \cdots, i_{p} \leqq m} \mu_{i_{1} \cdots i_{p}} X_{i_{1}} \cdots X_{i_{p}}, \mu_{i_{1}} \cdots i_{p} \in J . \mathfrak{F}_{p}$ is a vector space over $J$, and, in particular, $\mathfrak{F}_{0}=J$. For $a_{p}=\sum \mu_{i_{1}} \cdots i_{p} X_{i_{1}} \cdots X_{i_{p}} \in \mathfrak{F}_{p}$ and $b_{q}$ $=\sum \nu_{i_{p+1}} \cdots i_{p+q} X_{i_{p+1}} \cdots X_{i_{p+q}} \in \mathfrak{F}_{q}$, define

$$
a_{p} \cdot b_{q}=\sum \mu_{i_{1}} \cdots i_{p} \nu_{i_{p+1}} \cdots i_{p+q} X_{i_{1}} \cdots X_{i_{p+q}} .
$$

Thus the direct sum (in the strong sense)

$$
\Lambda=\Lambda\left(X_{1}, \cdots, X_{m} ; J\right)=\mathfrak{F}_{0}+\mathfrak{F}_{1}+\cdots+\mathfrak{F}_{p}+\cdots
$$

forms a ring with unit element 1 . Each element of $\Lambda$ is of the form $\sum_{p=0}^{\infty} a_{p}$, $a_{p} \in \mathfrak{F}_{p}$, and the multiplication is defined as follows:

$$
\sum a_{p} \cdot \sum b_{p}=\sum_{p=0}^{\infty} \sum_{i=0}^{p} a_{i} b_{p-i}, \quad a_{p}, b_{p} \in \mathfrak{F}_{p} .
$$

$\Lambda$ is called the (noncommutative formal) power series ring with indeterminates $X_{1}, \cdots, X_{m}$ over $J$.

The set $\mathfrak{D}=\mathfrak{F}_{1}+\mathfrak{F}_{2}+\cdots$ forms an ideal in $\Lambda$ and is called the fundamental ideal of $\Lambda$. It is easy to verify that $\mathfrak{D}^{p}=\mathfrak{F}_{p}+\mathfrak{F}_{p+1}+\ldots$. Define $\mathfrak{D}^{0}=\Lambda$. 
If $a=\sum a_{p}$ with $a \equiv 1 \bmod \mathfrak{D}$, i.e. $a_{0}=1$, then $a^{-1}$ exists. In fact, let $b=\sum b_{p}$ be such that

(1) $b_{0}=1, b_{1}=-a_{1}$;

(2) $b_{p}=-\left(a_{1} b_{p-1}+a_{2} b_{p-2}+\cdots+a_{p} b_{0}\right), p \geqq 2$.

Then $a \cdot b=1$.

Let $\Lambda^{\prime}$ be another power series ring with indeterminates $Y_{1}, \cdots, Y_{n}$ over $J$, and $\mathfrak{D}^{\prime}$ the fundamental ideal of $\Lambda^{\prime}$. A substitution $\bar{\sigma}: \Lambda \rightarrow \Lambda^{\prime}$ is a ring homomorphism defined as follows:

(a) $\bar{\sigma}(\mu)=\mu, \mu \in J$.

(b) $\bar{\sigma}\left(X_{i}\right) \equiv 0 \bmod \mathfrak{D}^{\prime}, i=1, \cdots, m$.

(c) Let $a=\sum_{p=0}^{\infty} a_{p}, a_{p} \in \mathfrak{F}_{p}$. Then each $\bar{\sigma}\left(a_{p}\right)=a_{p}^{(p)}+a_{p+1}^{(p)}+\cdots$ is well defined through $\bar{\sigma}\left(X_{i}\right)$. Define $\bar{\sigma}(a)=\sum_{p=0}^{\infty}\left(\sum_{i=0}^{p} a_{p}^{(i)}\right)$. It is not hard to verify that $\bar{\sigma}$ is a ring homomorphism, and $\bar{\sigma}\left(\mathfrak{D}^{p}\right) \subset \mathfrak{D}^{p}$.

If $\bar{\sigma}: \Lambda \rightarrow \Lambda^{\prime}$ and $\bar{\tau}: \Lambda^{\prime} \rightarrow \Lambda^{\prime \prime}$ are substitutions, so is $\bar{\tau} \bar{\sigma}: \Lambda \rightarrow \Lambda^{\prime \prime}$.

There is one and only one substitution $\bar{\sigma}: \Lambda \rightarrow \Lambda^{\prime}$ with preassigned $\bar{\sigma}\left(X_{i}\right)$ $\in \mathfrak{D}^{\prime}, i=1, \cdots, m$.

An invariant ideal $\Im$ of a power series ring $\Lambda$ over $J$ is defined as follows:

(1) $\Im=\Im(\Lambda)$ is an ideal of $\Lambda$ and is a function of $\Lambda$.

(2) For any substitution $\bar{\sigma}: \Lambda \rightarrow \Lambda^{\prime}$, we have $\bar{\sigma}(\Im(\Lambda)) \subset \Im\left(\Lambda^{\prime}\right)$.

5. Application of Magnus homomorphism in the presentation theory. To every free group $F$ with free generators $x_{1}, \cdots, x_{m}$, we associate a power series ring $\Lambda=\Lambda\left(X_{1}, \cdots, X_{m} ; J\right)$. Define a homomorphism $\phi: J F \rightarrow \Lambda$ such that $\phi\left(x_{i}\right)=1+X_{i}, i=1, \cdots, m$, and $\phi(\lambda)=\lambda, \lambda \in J$. The homomorphism $\phi$ is called the Magnus homomorphism of $J F$. It is clear that $\phi\left(x_{i}^{-1}\right)=1-X_{i}$ $+X_{i}^{2}-\cdots$. The Magnus homomorphism $\phi$ is linear if we regard $J F$ and $\Lambda$ as algebras over $J$.

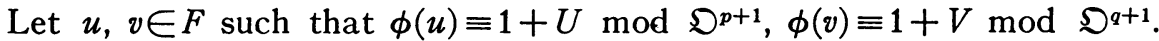
Then $\phi([u, v]) \equiv 1+[U, V] \bmod \mathfrak{D}^{p+q+1}$. It follows easily from this congruence identity that

$$
\phi\left(\left[x_{i_{1}}, \cdots, x_{i_{p}}\right]\right) \equiv 1+\left[X_{i_{1}}, \cdots, X_{i_{p}}\right] \bmod \mathfrak{D}^{p+1} .
$$

The homomorphism $\phi$ is due to Magnus [7]. His original definition is confined to the case when $J$ is the ring of all integers. However, the generalization to any ring $J$ with unit element is a trivial matter. It is known that $\phi$ maps the free group $F$ univalently into $\Lambda$. Witt has proved [9] that, for any $u \in F, \phi(u) \equiv 1 \bmod \mathfrak{D}^{p}$ if and only if $u$ belongs to the $p$ th lower central commutator subgroup $F_{p}$ of $F$.

Let $F^{\prime}$ be another free group with free generators $y_{1}, \cdots, y_{n}$, and $\phi^{\prime}: J F^{\prime} \rightarrow \Lambda^{\prime}=\Lambda\left(Y_{1}, \cdots, Y_{n} ; J\right)$ the Magnus homomorphism.

For any homomorphism $\sigma: J F \rightarrow J F^{\prime}$ extended from $\sigma: F \rightarrow F^{\prime}$, define a substitution $\bar{\sigma}: \Lambda \rightarrow \Lambda^{\prime}$ such that $\bar{\sigma}\left(X_{i}\right)=\phi^{\prime} \sigma\left(x_{i}\right)-1, i=1, \cdots, m$. Such a substitution $\bar{\sigma}$ exists and is unique. $\bar{\sigma}$ is said to be induced by $\sigma$. It is clear that $\bar{\sigma} \phi=\phi^{\prime} \sigma$. The following theorem is an immediate consequence of Lemma 2 . 
Theorem 3. Let $\mathfrak{M} / \mathfrak{N}$ be a quotient ring invariant. Then $\phi(\mathfrak{M}) / \phi(\mathfrak{N})$ is an algebraic invariant, and, for each presentation homomorphism $\sigma:\{F / R\}$ $\rightarrow\left\{F^{\prime} / R^{\prime}\right\}$, the associated homomorphism with respect to the invariant $\phi(\mathfrak{M}) / \phi(\mathfrak{N})$ is induced by the substitution $\bar{\sigma}: \Lambda \rightarrow \Lambda^{\prime}$.

CoRollaRy 1. If $M / N$ is a factor group invariant, then $\phi(J M) / \phi\left((N-1)_{M}\right)$ is an algebraic invariant.

COROLlaRY 2. If $\Im_{1}$ and $\Im_{2}$ are invariant ideals, then the $\left(\Im_{1}, \Im_{2}\right)$-filtration of $\phi(\mathfrak{M}) / \phi(\mathfrak{N})$ is also an algebraic invariant.

The proof of this corollary is essentially the same of that as Lemma 4.

EXAmple 1. Let $G=F / R$ be a finitely generated group. Any automorphism of $G$ may be given through a presentation isomorphism $\sigma:\{F / R\}$ $\cong\{F / R\} . \phi(J F) / \phi\left((R-1)_{F}\right)$ is an algebraic invariant, and so is its

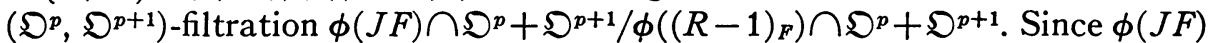
contains the vector space $\mathfrak{F}_{p}$ of all forms of degree $p$ for any $p \geqq 0$, we have $\phi(J F) \cap \mathfrak{D}^{p}+\mathfrak{D}^{p+1}=\mathfrak{D}^{p}$. The homomorphism $\sigma$ induces a substitution $\bar{\sigma}: \Lambda \rightarrow \Lambda$ with $\bar{\sigma}\left(X_{i}\right) \equiv \sum \lambda_{i j} X_{j} \bmod \mathfrak{D}^{2}$. According to the proof of Theorem 1 ,

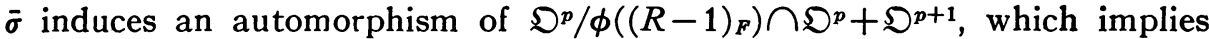

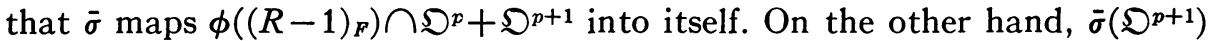

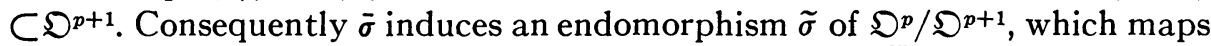

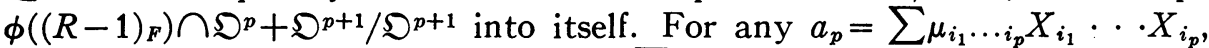
$\tilde{\sigma}\left(a_{p}+\mathfrak{D}^{p+1}\right)=\bar{\sigma}\left(a_{p}\right)+\mathfrak{D}^{p+1}=\sum \mu_{i_{1}} \cdots i_{p}\left(\sum \lambda_{i_{1} j} X_{j}\right) \cdots\left(\sum \lambda_{i_{p} j} X_{j}\right)+\mathfrak{D}^{p+1}$. The additive group of $\mathfrak{D}^{p} / \mathfrak{D}^{p+1}$ may be identified with $\mathfrak{F}_{p}$ by the correspondence

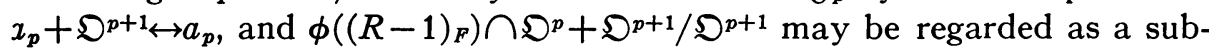
space $\Re_{p}$ of the vector space $\mathfrak{F}_{p}$. Thus we have

$$
\tilde{\sigma}\left(a_{p}\right)=\sum \mu_{i_{p}} \cdots i_{p}\left(\sum \lambda_{i_{1} j} X_{j}\right) \cdots\left(\sum \lambda_{i_{p} j} X_{j}\right),
$$

and $\tilde{\sigma}$ acts as a multilinear mapping of $\mathfrak{F}_{p}$ into itself. Moreover $\tilde{\sigma}\left(\Re_{p}\right) \subset \Re_{p}$, and $\tilde{\sigma}$ induces an automorphism of $\mathfrak{F}_{p} / \Re_{p}$. This is a generalization of a result due to Magnus, who considered the case with the following conditions: (a) $G /[G, G]$ is free abelian of rank $m$; (b) $F$ has exactly $m$ generators; (c) $p$ is the largest integer such that $R \subset F_{p}$; (d) $J$ is the ring of all integers.

ExAmple 2. Let $G$ be a group defined by two generators $x_{1}, x_{2}$ and a relation $r=\left[x_{1}, x_{2}, x_{1}, x_{1}\right] \equiv 1$, and let $G^{\prime}$ be a group defined by two generators $y_{1}, y_{2}$ and a relation $r^{\prime}=\left[y_{1}, y_{2}, y_{1}, y_{2}\right] \equiv 1$. We shall show that $G$ and $G^{\prime}$ are not isomorphic.

Suppose $G \cong G^{\prime}$. Let $F$ be the free group with free generators $x_{1}, x_{2}$, and $R$ the normal subgroup generated by $r$. Let $F^{\prime}$ be the free group with free generators $y_{1}, y_{2}$, and $R^{\prime}$ the normal subgroup generated by $r^{\prime}$. There is a presentation isomorphism $\sigma:\{F / R\} \cong\left\{F^{\prime} / R^{\prime}\right\}$. Let $\phi: J F \rightarrow \Lambda$ and $\phi^{\prime}: J F^{\prime} \rightarrow \Lambda^{\prime}$ be Magnus homomorphisms. 
For any $\{F / R\}$ with $R \subset[F, F]$, the factor group invariant

$$
[F, F] \cap R /[F, R]
$$

becomes $R /[F, R]$. In the cases of $G$ and $G^{\prime}$, we have $R \subset F_{4}, R^{\prime} \subset F_{4}^{\prime}$. Thus $\left(\mathfrak{D}^{4} \cap \phi(J R)+\mathfrak{D}^{5}\right) /\left(\mathfrak{O}^{4} \cap \phi\left(([R, F]-1)_{R}\right)+\mathfrak{D}^{5}\right)$

$$
\cong\left(\mathfrak{O}^{\prime 4} \cap \phi^{\prime}\left(J R^{\prime}\right)+\mathfrak{O}^{\prime 5}\right) /\left(\mathfrak{D}^{\prime 4} \cap \phi^{\prime}\left(\left(\left[R^{\prime}, F^{\prime}\right]-1\right)_{R^{\prime}}\right)+\mathfrak{O}^{\prime 5}\right),
$$

and this isomorphism is induced by a substitution $\bar{\sigma}: \Lambda \rightarrow \Lambda^{\prime}$. We take as $J$ the ring of all integers.

Since $[F, R] \subset F_{5}$, we have $\phi\left(([F, R]-1)_{R}\right) \subset \mathfrak{D}^{5}$, and, similarly,

$$
\phi^{\prime}\left(\left(\left[F^{\prime}, R^{\prime}\right]-1\right)_{R^{\prime}}\right) \subset \mathfrak{O}^{\prime 5} \text {. }
$$

It follows that

$$
\mathfrak{D}^{4} \cap \phi(J R)+\mathfrak{D}^{5} / \mathfrak{D}^{5} \cong \mathfrak{D}^{\prime 4} \cap \phi^{\prime}\left(J R^{\prime}\right)+\mathfrak{D}^{\prime 5} / \mathfrak{D}^{\prime 5} .
$$

Now $\phi\left(a r a^{-1}\right) \equiv \phi(r) \equiv 1+\left[X_{1}, X_{2}, X_{1}, X_{1}\right] \bmod \mathfrak{D}^{5}, a \in F$, and, for any $u \in R$, $\phi(u) \equiv 1+\mu\left[X_{1}, X_{2}, X_{1}, X_{1}\right] \bmod \mathfrak{D}^{5}, \mu \in J$. Consequently $\mathfrak{D}^{4} \cap \phi(J R)+\mathfrak{D}^{5} / \mathfrak{D}^{5}$ consists of all $\mu\left[X_{1}, X_{2}, X_{1}, X_{1}\right]+\mathfrak{S}^{5}, \mu \in J$. Similarly $\mathfrak{O}^{\prime 4} \cap \phi^{\prime}\left(J R^{\prime}\right)+\mathfrak{O}^{\prime 5} / \mathfrak{O}^{\prime 5}$ consists of all $\nu\left[Y_{1}, Y_{2}, Y_{1}, Y_{2}\right]+\mathfrak{D}^{\prime 5}, \nu \in J$. Thus $\bar{\sigma}\left(\left[X_{1}, X_{2}, X_{1}, X_{1}\right]\right)$ $\equiv \pm\left[Y_{1}, Y_{2}, Y_{1}, Y_{2}\right] \bmod \mathfrak{O}^{\prime 5}$. Let $\bar{\sigma}\left(X_{i}\right) \equiv \lambda_{i 1} Y_{1}+\lambda_{i 2} Y_{2} \bmod \mathfrak{O}^{\prime 2}, i=1,2$. We have $\bar{\sigma}\left(\left[X_{1}, X_{2}, X_{1}, X_{1}\right]\right) \equiv \sum \lambda_{1 i_{1}} \lambda_{2 i_{2}} \lambda_{1 i_{3}} \lambda_{1 i_{4}}\left[Y_{i_{1}}, Y_{i_{2}}, Y_{i_{3}}, Y_{i_{4}}\right] \bmod \mathfrak{D}^{\prime 5}$. Using the identities $\left[X_{i}, X_{j}\right]+\left[X_{j}, X_{i}\right]=0$ and $\left[X_{i}, X_{j}, X_{k}\right]+\left[\begin{array}{lll}\left.X_{j}, X_{k}, X_{i}\right] \\ X_{j}\end{array}\right.$ $+\left[X_{k}, X_{i}, X_{j}\right]=0$ we obtain $\bar{\sigma}\left(\left[X_{1}, X_{2}, X_{1}, X_{1}\right]\right) \equiv \Delta \lambda_{11}^{2}\left[Y_{1}, Y_{2}, Y_{1}, Y_{1}\right]$ $+2 \Delta \lambda_{11} \lambda_{12}\left[Y_{1}, Y_{2}, Y_{1}, Y_{2}\right]+\Delta \lambda_{12}^{2}\left[Y_{1}, Y_{2}, Y_{2}, Y_{2}\right] \bmod \mathcal{O}^{\prime 5}$, where $\Delta=\lambda_{11} \lambda_{22}$ $-\lambda_{12} \lambda_{21}$. Thus $\pm\left[Y_{1}, Y_{2}, Y_{1}, Y_{2}\right]=\Delta\left(\lambda_{11}^{2}\left[Y_{1}, Y_{2}, Y_{1}, Y_{1}\right]+2 \lambda_{11} \lambda_{12}\left[Y_{1}, Y_{2}, Y_{1}, Y_{2}\right]\right.$ $\left.+\lambda_{12}^{2}\left[Y_{1}, Y_{2}, Y_{2}, Y_{2}\right]\right)$. It is easy to verify that

$$
\left[Y_{1}, Y_{2}, Y_{1}, Y_{1}\right], \quad\left[Y_{1}, Y_{2}, Y_{1}, Y_{2}\right], \quad\left[Y_{1}, Y_{2}, Y_{2}, Y_{2}\right]
$$

are linearly independent. According to the above equality, it is necessary that $\Delta \neq 0, \lambda_{11}=0, \lambda_{12}=0$. However, $\lambda_{11}=0$ and $\lambda_{12}=0$ lead to the contradiction $\Delta=0$. Hence our assertion is proved.

6. $B$-presentations. We shall strengthen the conception of isomorphism as follows: Let $B$ be a fixed group. Consider pairs $\left(G, \alpha_{0}\right)$, where $G$ is a group, and $\alpha_{0}: G \rightarrow B$ is a homomorphism. $\left(G, \alpha_{0}\right)$ and $\left(G^{\prime}, \alpha_{0}^{\prime}\right)$ are said to be $B$-isomorphic, $\left(G, \alpha_{0}\right) \cong{ }^{B}\left(G^{\prime}, \alpha_{0}^{\prime}\right)$, if there is an isomorphism-onto $\rho: G \cong G^{\prime}$ with $\alpha_{0}^{\prime} \rho=\alpha_{0}$. Since $\left(G, \alpha_{0}\right) \cong{ }^{B}\left(G^{\prime}, \alpha_{0}^{\prime}\right)$ implies $G \cong G^{\prime}, B$-isomorphism is in a sense stronger than isomorphism. The definition of $B$-isomorphism includes isomorphism as a particular case by taking as $B$ the trivial group $\{e\}$.

A $B$-presentation is a triple $\{F / R, \alpha\}$, where $R$ is a normal subgroup of a free group $F$, and $\alpha: F \rightarrow B$ is a homomorphism with a kernel containing $R$. By a $B$-presentation homomorphism $\sigma:\{F / R, \alpha\} \rightarrow\left\{F^{\prime} y R^{\prime}, \alpha^{\prime}\right\}$, we mean a presentation homomorphism $\sigma:\{F / R\} \rightarrow\left\{F^{\prime} / R^{\prime}\right\}$ with $\alpha^{\prime} \sigma=\alpha$. If, in addi- 
tion, $\sigma$ is a presentation isomorphism, then $\sigma$ is called a $B$-presentation isomorphism. A $B$-presentation homomorphism $\tau:\{F / R, \alpha\} \rightarrow\left\{F^{\prime} / R^{\prime}, \alpha^{\prime}\right\}$ is said to be equivalent to $\sigma$, if $\sigma$ and $\tau$, taken as presentation homomorphisms, are equivalent, i.e. $\sigma$ and $\tau$ induce the same homomorphism $F / R \rightarrow F^{\prime} / R^{\prime}$.

Lemma 5. Let $\{F / R, \alpha\}$ and $\left\{F^{\prime} / R^{\prime}, \alpha^{\prime}\right\}$ be B-presentations, and $\alpha_{0}: F / R$ $\rightarrow B, \alpha_{0}^{\prime}: F^{\prime} / R^{\prime} \rightarrow B$, the homomorphisms induced by $\alpha$ and $\alpha^{\prime}$, respectively. Given a homomorphism $\rho: F / R \rightarrow F^{\prime} / R^{\prime}, \alpha_{0}^{\prime} \rho=\alpha_{0}$, we can always construct $\sigma:\{F / R, \alpha\} \rightarrow\left\{F^{\prime} / R^{\prime}, \alpha^{\prime}\right\}$ such that $\rho$ is induced by $\sigma$.

Proof. According to Lemma $3, \rho$ is induced by a presentation homomorphism $\sigma:\{F / R\} \rightarrow\left\{F^{\prime} / R^{\prime}\right\} . \quad \alpha^{\prime} \sigma(u)=\alpha_{0}^{\prime}[\sigma(u) \cdot R]=\alpha_{0}^{\prime} \rho(u R)=\alpha_{0}(u R)$ $=\alpha(u), u \in F$. Hence $\sigma$ is a $B$-presentation homomorphism.

Two $B$-presentations $\{F / R, \alpha\}$ and $\left\{F^{\prime} / R^{\prime}, \alpha^{\prime}\right\}$ are isomorphic: $\{F / R, \alpha\}$ $\cong\left\{F^{\prime} / R^{\prime}, \alpha^{\prime}\right\}$, if and only if there is an isomorphism $\rho: F / R \cong F^{\prime} / R^{\prime}$ with $\alpha_{0}^{\prime} \rho=\alpha_{0}$.

The theory of $B$-presentations also includes the theory of presentations as a special case by taking as $B$ the trivial group $\{e\}$.

We shall give a motivation of the conception of $B$-isomorphism. An ordered and oriented $n$-link $L=L_{1} \cup \ldots \cup L_{n}$ is the union of $n$ disjoint, oriented, simple closed curves in euclidean 3-space $E$. Each $L_{i}$ is called the $i$ th component. Let $L^{\prime}=L_{1}^{\prime} \cup \ldots \cup L_{n}^{\prime}$ be another ordered and oriented $n$-link. Then $L$ and $L^{\prime}$ are said to be equivalent if there is a homeomorphism $\chi: E \rightarrow E$ such that the $i$ th component of $L$ is mapped by $\chi$ onto the $i$ th component of $L^{\prime}$ with orientation preserved. Let $G=\pi_{1}(E-L)$ and $G^{\prime}$ $=\pi_{1}\left(E-L^{\prime}\right)$ be the groups of the links $L$ and $L^{\prime}$ respectively, and let $B$ be a free abelian group with basis $\left\{t_{1}, t_{2}, \cdots, t_{n}\right\} . \chi$ induces an isomorphism $\rho: G \rightarrow G^{\prime}$. For each $u \in G$, denote by $\lambda_{i}(u)$ the linking number of $L_{i}$ with a closed path representing $u$. Then $\alpha_{0}: G \rightarrow B$ defined by $\alpha_{0}(u)=t_{1}^{\lambda_{1}(u)} \cdots t_{n}^{\lambda_{n}(u)}$ is a homomorphism-onto with $[G, G]$ as kernel. Similarly, define $\alpha_{0}^{\prime}: G^{\prime} \rightarrow B$. Thus $\left(G, \alpha_{0}\right) \cong^{B}\left(G^{\prime}, \alpha_{0}^{\prime}\right)$, and the equivalence of two ordered and oriented links implies a $B$-isomorphism of their groups.

7. Application of free differentiation of Fox in $B$-presentation theory. Let $J$ be a ring with unit element, and $F$ a free group with free generators $x_{1}, \cdots, x_{m}$. R. H. Fox has defined [4] operations $\partial / \partial x_{j}$ in $J F$ as follows: Let $w=a_{0} x_{j}^{\epsilon_{1}} a_{1} \cdots x_{j}^{\epsilon_{k}} a_{k}, \epsilon_{i}= \pm 1$, be a word, where $a_{0}, \cdots, a_{k}$ are words not involving $x_{j}$. Then $\partial u / \partial x_{j}=\sum_{i=1}^{k} \epsilon_{i} u_{i}, u_{i}=a_{0} x_{j}^{\epsilon_{1}} a_{1} \cdots a_{i-1} x_{j}^{\left.\epsilon_{i}-1\right) / 2}$. Each operation $\partial / \partial x_{j}$ is then extended to the group ring $J F$ by linearity. Let $o: J F \rightarrow J$ be coefficient homomorphism as defined in $\$ 1$. The identity

$$
u=o(u)+\sum \frac{\partial u}{\partial x_{i}}\left(x_{i}-1\right), \quad u \in J F,
$$

holds. It is not hard to verify that $\sum v_{i}\left(x_{i}-1\right)=0, v_{i} \in J F$, implies $v_{i}=0$. Thus the above identity is unique, i.e., for any $u \in J F$, 


$$
u=o(u)+\sum \bar{u}_{i}\left(x_{i}-1\right)
$$

implies $\bar{u}_{i}=\partial u / \partial x_{i}$. Furthermore

$$
\begin{aligned}
u & =o(u)+\sum\left[o\left(\frac{\partial u}{\partial x_{i}}\right)+\sum \frac{\partial^{2} u}{\partial x_{j} \partial x_{i}}\left(x_{j}-1\right)\right]\left(x_{i}-1\right) \\
& =o(u)+\sum o\left(\frac{\partial u}{\partial x_{i}}\right)\left(x_{i}-1\right)+\sum \frac{\partial^{2} u}{\partial x_{j} \partial x_{i}}\left(x_{j}-1\right)\left(x_{i}-1\right) .
\end{aligned}
$$

In general we have

$$
\begin{aligned}
u=o(u) & +\sum_{k=1}^{p-1} \sum o\left(\frac{\partial^{k} u}{\partial x_{i_{1}} \cdots \partial x_{i_{k}}}\right)\left(x_{i_{1}}-1\right) \cdots\left(x_{i_{k}}-1\right) \\
& +\sum \frac{\partial^{p} u}{\partial x_{i_{1}} \cdots \partial x_{i_{p}}}\left(x_{i_{1}}-1\right) \cdots\left(x_{i_{p}}-1\right),
\end{aligned}
$$

and the expansion is unique. The Magnus homomorphism $\phi: J F \rightarrow \Lambda$ may be explicitly given by

$$
\phi(u)=o(u)+\cdots+\sum o\left(\frac{\partial^{p} u}{\partial x_{i_{1}} \cdots \partial x_{i_{p}}}\right) X_{i_{1}} \cdots X_{i_{p}}+\cdots .
$$

Let $O$ be the fundamental ideal of $J F$ and thus the kernel of $o: J F \rightarrow J$. It follows that $O=\phi^{-1}(\mathfrak{D})$. Therefore $O^{p} \subset \phi^{-1}\left(\mathfrak{D}^{p}\right)$. Due to (1) and (2), $u \in \phi^{-1}\left(\mathfrak{D}^{p}\right)$ implies

$$
u=\sum \frac{\partial^{p} u}{\partial x_{i_{1}} \cdots \partial x_{i_{p}}}\left(x_{i_{1}}-1\right) \cdots\left(x_{i_{p}}-1\right)
$$

which belongs to $O^{p}$. We have $O^{p}=\phi^{-1}\left(\mathfrak{D}^{p}\right)$. Hence each $u \in O^{p}$ may be uniquely expressed as $u=\sum u_{i_{1} \cdots_{i_{p}}}\left(x_{i_{1}}-1\right) \cdots\left(x_{i_{p}}-1\right), u_{i_{1} \cdots_{i_{p}}} \in J F$, and $O^{p}$ is a left vector space over $J F$ with all $\left(x_{i_{1}}-1\right) \cdots\left(x_{i_{p}}-1\right)$ as basis.

For $O^{p}$ we construct a vector space $\left[O^{p}\right]_{B}$ which consists of the totality of forms $\sum b_{i_{1} \cdots i_{p}} X_{i_{1}} \cdots X_{i_{p}}, b_{i_{1}} \cdots i_{p} \in J B$, of degree $p$ in noncommutative indeterminants $X_{1}, \cdots, X_{m}$ over the ring $J B$.

To a $B$-presentation $\{F / R, \alpha\}$, there is associated a linear mapping $\alpha_{p}: O^{p} \rightarrow\left[O^{p}\right]_{B}$ with $\alpha_{p}(u)=\sum \alpha\left(\partial^{p} u / \partial x_{i_{1}} \cdots \partial x_{i_{p}}\right) X_{i_{1}} \cdots X_{i_{p}}, u \in O^{p}$. If $\alpha$ is onto, so is $\alpha_{p} . \alpha_{p}$ is called the $p$ th linear mapping induced by $\alpha$.

Let $\left\{F^{\prime} / R^{\prime}, \alpha^{\prime}\right\}$ be another $B$-presentation, where $F^{\prime}$ has free generators $y_{1}, \cdots, y_{n}$. Denote $O^{\prime}$ the fundamental ideal of $J F^{\prime}$ and by $\alpha_{p}^{\prime}: O^{\prime p} \rightarrow\left[O^{\prime p}\right]_{B}$ the $p$ th linear mapping induced by $\alpha^{\prime} . \sigma:\{F / R, \alpha\} \rightarrow\left\{F^{\prime} / R^{\prime}, \alpha^{\prime}\right\}$ is a $B$-presentation homomorphism and is extended to $\sigma: J F \rightarrow J F^{\prime}$. For the extended homomorphism $\alpha: J F \rightarrow J B, \alpha^{\prime}: J F^{\prime} \rightarrow J B^{\prime}$, it is again true that $\alpha^{\prime} \sigma=\alpha$. The linear mapping

$$
\tilde{\sigma}_{p}:\left[O^{p}\right]_{B} \rightarrow\left[O^{\prime p}\right]_{B}
$$


defined by $\tilde{\sigma}_{p}\left(X_{i_{1}} \cdots X_{i_{p}}\right)=\alpha_{p}^{\prime} \sigma\left(\left(x_{i_{1}}-1\right) \cdots\left(x_{i_{p}}-1\right)\right)$ is called the $p$ th linear mapping induced by $\sigma$. For any $u \in O^{p}$,

$$
\begin{aligned}
\tilde{\sigma}_{p} \alpha_{p}(u) & =\tilde{\sigma}_{p}\left(\sum \alpha\left(\frac{\partial^{p} u}{\partial x_{i_{1}} \cdots \partial x_{i_{p}}}\right) X_{i_{1}} \cdots X_{i_{p}}\right) \\
& =\sum \alpha\left(\frac{\partial^{p} u}{\partial x_{i_{1}} \cdots \partial x_{i_{p}}}\right) \tilde{\sigma}_{p}\left(X_{i_{1}} \cdots X_{i_{p}}\right) \\
& =\sum \alpha^{\prime} \sigma\left(\frac{\partial^{p} u}{\partial x_{i_{1}} \cdots \partial x_{i_{p}}}\right) \alpha_{p}^{\prime} \sigma\left(\left(x_{i_{1}}-1\right) \cdots\left(x_{i_{p}}-1\right)\right) .
\end{aligned}
$$

Since $\sigma\left(\left(x_{i}-1\right) \cdots\left(x_{i_{p}}-1\right)\right) \in O^{\prime p}$,

$$
\begin{aligned}
\tilde{\sigma}_{p} \alpha_{p}(u) & =\alpha_{p}^{\prime}\left(\sum \sigma\left(\frac{\partial^{p} u}{\partial x_{i_{1}} \cdots \partial x_{i_{p}}}\right) \sigma\left(\left(x_{i_{1}}-1\right) \cdots\left(x_{i_{p}}-1\right)\right)\right) \\
& =\alpha_{p}^{\prime} \sigma(u) .
\end{aligned}
$$

Consequently, $\tilde{\sigma}_{p} \alpha_{p}=\alpha_{p}^{\prime} \sigma$.

The following theorem is a direct consequence of Lemma 2.

Theorem 4. Let $\mathfrak{M} / \mathfrak{N}$ be a quotient ring invariant, and $\sigma:\{F / R, \alpha\}$ $\rightarrow\left\{F^{\prime} / R^{\prime}, \alpha^{\prime}\right\}$ a B-presentation homomorphism. Then there is a homomorphism

$$
\tilde{\sigma}_{p}^{*}: \frac{\alpha_{p}\left(O^{p} \cap \mathfrak{M}(F, R)\right)}{\alpha_{p}\left(O^{p} \cap \mathfrak{R}(F, R)\right)} \rightarrow \frac{\alpha_{p}^{\prime}\left(O^{\prime p} \cap \mathfrak{M}\left(F^{\prime}, R^{\prime}\right)\right)}{\alpha_{p}^{\prime}\left(O^{\prime} p \cap \mathfrak{N}\left(F^{\prime}, R^{\prime}\right)\right)}
$$

induced by $\widetilde{\sigma}_{p}:\left[O^{p}\right]_{B} \rightarrow\left[O^{\prime p}\right]_{B}$ such that $\tilde{\sigma}_{p}^{*}$ does not change when $\sigma$ is replaced by an equivalent $B$-presentation homomorphism.

CoRollary 1. If $\sigma$ is a B-presentation isomorphism, then $\tilde{\sigma}_{p}^{*}$ is an isomorphism-onto.

COROLlary 2. If $\sigma$ is a B-presentation isomorphism, and $\alpha$ is onto, then

$$
\left[O^{p}\right]_{B} / \alpha_{p}\left(O^{p} \cap(R-1)_{F}\right) \cong\left[O^{\prime p}\right]_{B} / \alpha_{p}^{\prime}\left(O^{\prime p} \cap\left(R^{\prime}-1\right)_{F^{\prime}}\right) .
$$

Proof. Take $\mathfrak{M} / \mathfrak{R}=J F /(R-1)_{F}$. Then $\alpha_{p}\left(O^{p} \cap J F\right)=\alpha_{p}\left(O^{p}\right)=\left[O^{p}\right]_{B}$.

The $J B$-module $\alpha_{p}\left(O^{p} \cap \mathfrak{M}\right) / \alpha_{p}\left(O^{p} \cap \mathfrak{N}\right)$ is, so to speak, an algebraic invariant for $B$-presentations. We do not intend to give explicit definitions of such kind.

We shall consider those $B$-presentations $\{F / R, \alpha\}$ such that $\alpha$ is onto, and $B$ is a cyclic group. Furthermore take as $J$ a field. Then $J B$ is a principal ideal ring.

Suppose that $F$ has $x_{1}, \cdots, x_{m}$ as free generators. $\left[O^{p}\right]_{B}$ is then a vector space with all $X_{i_{1}} \cdots X_{i_{p}}$ as basis over $J B .\left[O^{p}\right]_{B} / \alpha_{p}\left((R-1)_{F} \cap O^{p}\right)$ is characterized by its elementary divisors. 
In particular, let $p=1$ and let $R$ be generated by a finite number of elements $r_{1}, \cdots, r_{k}$. Then $(R-1)_{F} \cap O=(R-1)_{F}$ is an ideal generated by all

$$
r_{i}-1=\sum\left(\partial r_{i} / \partial x_{j}\right)\left(x_{j}-1\right) .
$$

$\alpha_{1}\left(r_{i}-1\right)=\sum \alpha\left(\partial r_{i} / \partial x_{j}\right) X_{j}$. For any $a \in J F$, it follows from $a\left(r_{i}-1\right)$ $=\sum a\left(\partial r_{i} / \partial x_{j}\right)\left(x_{j}-1\right)$ that

$$
\alpha_{1}\left(a\left(r_{i}-1\right)\right)=\alpha(a) \cdot \alpha_{1}\left(r_{i}-1\right) .
$$

On the other hand

$$
\begin{aligned}
\alpha_{1}\left(\left(r_{i}-1\right) a\right) & =\alpha_{1}\left[\sum\left(\partial\left(r_{i}-1\right) / \partial x_{j} \cdot o(a)+\left(r_{i}-1\right) \partial a / \partial x_{j}\right)\left(x_{j}-1\right)\right] \\
& =o(a) \cdot \sum \alpha\left(\partial r_{i} / \partial x_{j}\right) X_{j}=o(a) \alpha_{1}\left(r_{i}-1\right) .
\end{aligned}
$$

Thus $\alpha_{1}\left((R-1)_{F}\right)$, as a vector space, is generated by all $\sum \alpha\left(\partial r_{i} / \partial x_{j}\right) X_{j}$. Then column deficiency of the matrix $\left\|\alpha\left(\partial r_{i} / \partial x_{j}\right)\right\|$ is the Betti number of $[O]_{B} / \alpha_{1}\left((R-1)_{F}\right)$, and the elementary divisors of the matrix, which are neither zero nor units, are the torsion numbers of $[O]_{B} / \alpha_{1}\left((R-1)_{F}\right)$. If two $B$-presentations are isomorphic, then the Betti numbers must be identical and each corresponding pair of the torsion numbers only differ up to a unit in $J B$.

In the case that $B$ is infinite cyclic, the product of all torsion numbers is a polynomial in a generator of $B$ with coefficients in the field $J$. This polynomial differs from the Alexander polynomial $[1 ; 8]$ only by the coefficient domain. The latter has integral coefficients. The computation of the torsion numbers and the Alexander polynomial by using the Jacobian matrix $\left|\alpha\left(\partial r_{i} / \partial x_{j}\right)\right|$ is due to R. H. Fox [4].

REMARK. Theorem 4 and its corollaries will remain true if the indeterminates $X_{1}, \cdots, X_{m}$ are commutative for each $\left[O^{p}\right]_{B}$. The new $\left[O^{p}\right]_{B}$ will consist of all forms $\sum b_{i_{1} \cdots i_{p}} X_{i_{1}} \cdots X_{i_{p}}$, where $b_{i_{1} \cdots i_{p}} \in J B$ and $X_{i_{1}} \cdots X_{i_{p}}=X_{j_{1}} \cdots X_{j_{p}}$ for any permutation $\left(j_{1}, \cdots, j_{p}\right)$ of $\left(i_{1}, \cdots, i_{p}\right)$.

ExAMPLE. Let $G$ be a group defined by two generators $x_{1}, x_{2}$ and two relations $x_{1}^{4} \equiv 1, x_{1} x_{2} x_{1} \equiv x_{2} x_{1} x_{2}$, and let $G^{\prime}$ be a group defined by two generators $y_{1}, y_{2}$ and two relations $y_{1}^{4} \equiv 1, y_{2}\left[y_{1}^{-1}, y_{2}^{-1}\right] \equiv\left[y_{1}, y_{2}\right] y_{1}$. We shall show that $G$ and $G^{\prime}$ are not isomorphic.

Let $F$ be the free group with free generators $x_{1}, x_{2}$, and $R$ the normal subgroup generated by $x_{1}^{4}$ and $r=x_{1} x_{2} x_{1}\left(x_{2} x_{1} x_{2}\right)^{-1}$. Let $F^{\prime}$ be the free group with free generators $y_{1}, y_{2}$, and $R^{\prime}$ the normal subgroup generated by $y_{1}^{4}$ and $r^{\prime}=y_{2}\left[y_{1}^{-1}, y_{2}^{-1}\right]\left(\left[y_{1}, y_{2}\right] y_{1}\right)^{-1}$. Then $G=F / R$ and $G^{\prime}=F^{\prime} / R^{\prime}$.

Let $B$ be a cyclic group of order 4 and consist of elements $1, t, t^{2}, t^{3}$. Observe that both $G /[G, G]$ and $G^{\prime} /\left[G^{\prime}, G^{\prime}\right]$ are cyclic of order 4 , and an isomorphism-onto $\rho: G \cong G^{\prime}$ would induce $G /[G, G] \cong G^{\prime} /\left[G^{\prime}, G^{\prime}\right]$. Define by $\alpha_{0}^{\prime}: G^{\prime} \rightarrow B$ the homomorphism-onto such that $\alpha_{0}^{\prime}\left(y_{1} R^{\prime}\right)=t$, and $\left[G^{\prime}, G^{\prime}\right]$ is the kernel. Then $\left(G, \alpha_{0}^{\prime} \rho\right) \cong{ }^{B}\left(G^{\prime}, \alpha_{0}^{\prime}\right) . \alpha_{0}^{\prime} \rho: G \rightarrow B$ has $[G, G]$ as its kernel. There are only two possible homomorphisms which can be $\alpha_{0}^{\prime} \rho$, namely: 


$$
\begin{array}{lll}
\alpha_{0}: G \rightarrow B & \text { with } & \alpha_{0}\left(x_{1} R\right)=t, \\
\beta_{0}: G \rightarrow B & \text { with } & \beta_{0}\left(x_{1} R\right)=t^{3} .
\end{array}
$$

Consequently $G \cong G^{\prime}$ implies either $\left(G, \alpha_{0}\right) \cong{ }^{B}\left(G^{\prime}, \alpha_{0}^{\prime}\right)$ or $\left(G, \beta_{0}\right) \cong{ }^{B}\left(G^{\prime}, \alpha_{0}^{\prime}\right)$.

Define homomorphisms $\alpha: F \rightarrow B$ with kernel $R \cdot[F, F]$ and $\alpha\left(x_{1}\right)=t$; $\beta: F \rightarrow B$ with kernel $R \cdot[F, F]$ and $\beta\left(x_{1}\right)=t^{3} ; \alpha^{\prime}: F^{\prime} \rightarrow B$ with kernel $R^{\prime} \cdot\left[F^{\prime}, F^{\prime}\right]$ and $\alpha^{\prime}\left(y_{1}\right)=t$. Then $G \cong G^{\prime}$ would imply either

$$
\{F / R, \alpha\} \cong\left\{F^{\prime} / R^{\prime}, \alpha^{\prime}\right\} \quad \text { or } \quad\{F / R, \beta\} \cong\left\{F^{\prime} / R^{\prime}, \alpha^{\prime}\right\} .
$$

We take as $J$ the field of all integers modulo 3. By straightforward computation, we find the torsion numbers of

$$
\begin{aligned}
& {[O]_{B} / \alpha_{1}\left((R-1)_{F}\right),} \\
& {[O]_{B} / \beta_{1}\left((R-1)_{F}\right),} \\
& {\left[O^{\prime}\right]_{B} / \alpha_{1}^{\prime}\left(\left(R^{\prime}-1\right)_{F^{\prime}}\right),}
\end{aligned}
$$

respectively as follows:

$$
\begin{array}{ll}
1+t+t^{2}+t^{3}, & 1+t \\
1+t+t^{2}+t^{3}, & 1+t \\
1+t+t^{2}+t^{3}, & 1+t^{2}
\end{array}
$$

$1+t^{2}$ is not divisible by $1+t$ in $J B$. Hence $G$ and $G^{\prime}$ are not isomorphic.

\section{Bibliography}

1. J. W. Alexander, Topological invariants of knots and links, Trans. Amer. Math. Soc. vol. 30 (1928) pp. 275-306.

2. R. Baer, Representation of groups as quotient groups. I, Trans. Amer. Math. Soc. vol. 58 (1945) pp. 295-347.

3. S. Eilenberg and S. MacLane, General theory of natural equivalence, Trans. Amer. Math. Soc. vol. 58 (1945) pp. 231-294.

4. R. H. Fox, Free differential calculus, I, Ann. of Math. vol. 57 (1953) pp. 547-560.

5. H. Hopf, Fundamentalgruppe und zweite Bettische Gruppe, Comment. Math. Helv. vol. 14 (1941-42) pp. 257-309.

6. R. C. Lyndon, Cohomology theory of groups with a single defining relation, Ann. of Math. vol. 52 (1950) pp. 650-665.

7. W. Magnus, Bezeihungen zwischen Gruppen und Idealen in einem speziellen Ring, Math. Ann. vol. 111 (1935) pp. 259-280.

8. K. Reidemeister, Einführung in die kombinatorische Topologie, Braunschweig, Vieweg, 1932.

9. E. Witt, Treue Darstellung der Liescher Ringe, J. Reine Angew. Math. vol. 177 (1937) pp. $152-160$.

UNIVERSITY OF ILLINOIS,

URBANA, ILL.

UNIVERSITY OF HONG Kong,

HoNG Kong. 